\title{
Searches for additional Higgs bosons with the CMS detector
}

\author{
, Dermot Moran ${ }^{a, 1, *}$ \\ ${ }^{a}$ CIEMAT, \\ Av. Complutense, 40, 28040 Madrid, Spain \\ E-mail: dermot.anthony.moran@cern.ch
}

Searches for additional Higgs bosons with the CMS detector at the LHC are presented. The analyses are based on proton-proton collisions at $\sqrt{s}=13 \mathrm{TeV}$ recorded in 2016, corresponding to an integrated luminosity of $35.9 \mathrm{fb}^{-1}$. The observed data are consistent with the standard model expectations. Upper limits on the Higgs boson production cross section times branching ratio are set for each search. These results further reduce the allowed parameter space for extensions of the standard model.

40th International Conference on High Energy physics - ICHEP2020

July 28 - August 6, 2020

Prague, Czech Republic (virtual meeting)

\footnotetext{
${ }^{1}$ On behalf of the CMS Collaboration.

${ }^{*}$ Speaker
} 


\section{Introduction}

The discovery of the standard model (SM) Higgs boson with a mass close to $125 \mathrm{GeV}$ [13], denoted here as $\mathrm{h}(125)$, has led to a new era in particle physics. In order to determine whether the SM gives a complete description of the Higgs sector, precise measurements of the $\mathrm{h}(125)$ coupling strengths, CP structure and kinematic distributions are required. A complementary and important strategy involves the search for additional Higgs bosons whose existence would prove the presence of beyond-the-SM (BSM) physics in the form of a non minimal Higgs sector. The search for additional scalar resonances remains one of the main objectives at the LHC.

One of the simplest extensions of the SM Higgs sector is known as the two-Higgsdoublet-model (2HDM) [4] and involves the introduction of a second scalar doublet. Various formulations of the $2 \mathrm{HDM}$ predict different couplings of the two doublets to right-handed quarks and charged leptons. There are five physical Higgs bosons predicted: two CP-even neutral bosons $\mathrm{h}$ and $\mathrm{H}$; a neutral CP-odd boson $\mathrm{A}$; and two charged bosons $\mathrm{H}^{ \pm}$. In most formulations of the $2 \mathrm{HDM}$, h corresponds to the $\mathrm{h}(125)$, and $\mathrm{H}$ is an additional heavy CPeven Higgs boson. The 2HDM has two important free parameters, $\alpha$ and $\tan \beta$, which are the mixing angle and the ratio of the vacuum expectation values of the two Higgs doublets, respectively. In the alignment limit, which occurs at $\cos (\beta-\alpha)=0$, the properties of $h$ approach those of the SM Higgs boson.

In this report, searches for additional neutral Higgs bosons by the CMS experiment at the LHC [6], covering a number of different decay channels and production mechanisms, are presented. The analyses are based on proton-proton collisions at $\sqrt{s}=13 \mathrm{TeV}$ recorded in 2016, corresponding to an integrated luminosity of $35.9 \mathrm{fb}^{-1}$.

\section{Search for light $\mathrm{H}^{ \pm} \rightarrow \mathrm{cs}$}

At low values of $\tan \beta$ the dominant decay of a light charged Higgs boson is into a charm and a strange quark. A search is performed for $\mathrm{H}^{ \pm} \rightarrow \mathrm{cs}$ in the process of top quark pair production, where one top quark decays to a bottom quark and a charged Higgs boson, and the other to a bottom quark and a $\mathrm{W}$ boson [7].

The semileptonic final state of interest comprises an isolated lepton, missing transverse momentum, and at least four jets, of which two are tagged as b jets. The invariant mass of the two highest $\mathrm{p}_{\mathrm{T}}$ non-b jets $\left(m_{j j}\right)$ is used as the final discriminant variable for signal extraction. Furthermore, the events are categorized based on the charm tagging of these two jets. To help identify t $\bar{t}$ events, a kinematic fit is performed on the reconstructed objects using top quark mass constraints.

The dominant background process from SM t $\bar{t}$ is estimated from next-to-leading order (NLO) simulation. The observed and predicted number of events from SM processes are in agreement within uncertainties. Figure 1 (left) shows the resulting upper limits placed on $\mathrm{B}\left(\mathrm{t} \rightarrow \mathrm{H}^{ \pm} \mathrm{b}\right)$ assuming $\mathrm{B}\left(\mathrm{H}^{ \pm} \rightarrow \mathrm{cs}\right)=100 \%$. 

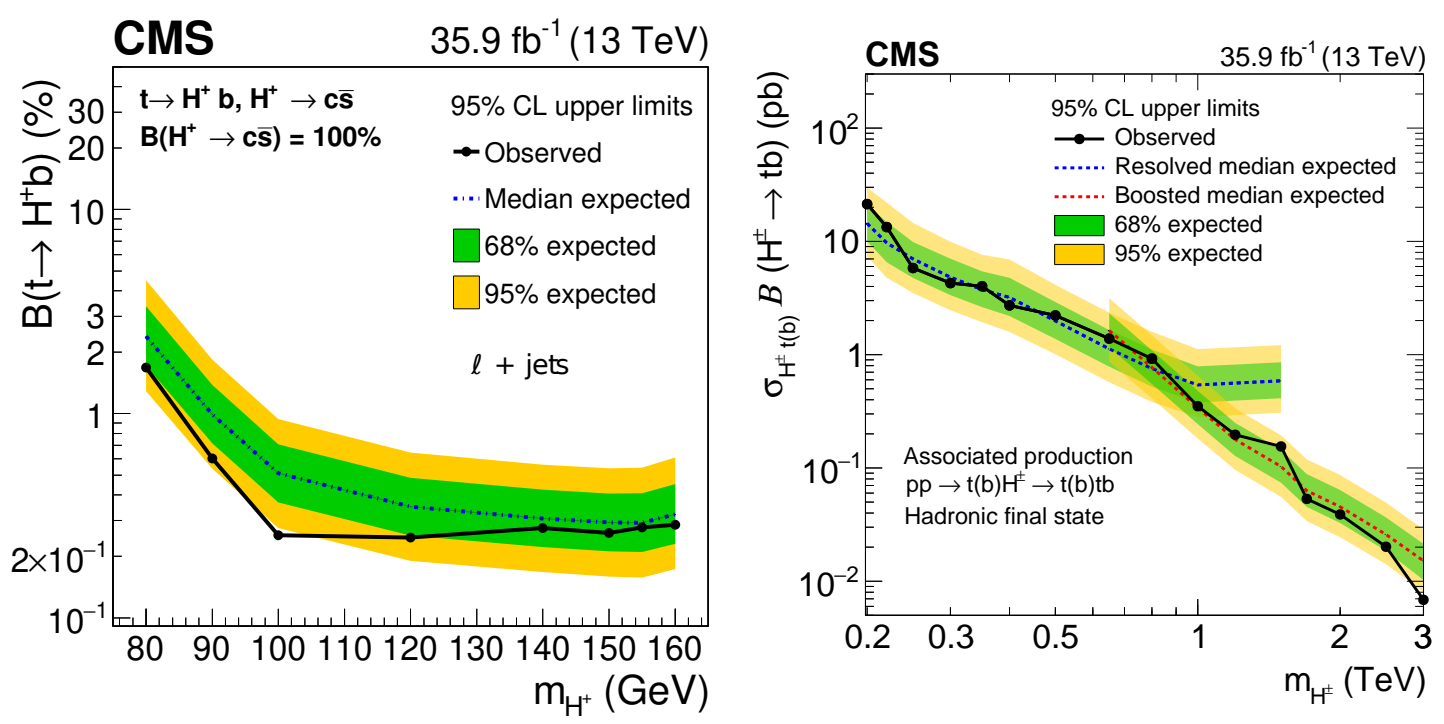

Figure 1: Upper limits on $\mathrm{B}\left(\mathrm{t} \rightarrow \mathrm{H}^{ \pm} \mathrm{b}\right)$ assuming $\mathrm{B}\left(\mathrm{H}^{ \pm} \rightarrow \mathrm{cs}\right)=100 \%$ (left) [7], and upper limits on the product of $\sigma_{\mathrm{H}^{ \pm} t(b)}$ and $B\left(H^{ \pm} \rightarrow t b\right)$ (right) [9].

\section{Search for heavy $\mathrm{H}^{ \pm} \rightarrow \mathrm{tb}$}

A search is performed for a charged Higgs boson decaying into a top and a bottom quark in the all-jet final state [9]. The production of the boson in association with a top quark and direct production via an s-channel process are considered.

Two distinct event topologies are targeted, boosted and resolved. In the resolved case, a top quark candidate can be reconstructed from two jets associated with a $\mathrm{W}$ boson decay and one jet identified as originating from a $\mathrm{b}$ quark. The invariant mass $m_{t b}$ of the $\mathrm{H}^{ \pm}$ candidate is used as the final discriminating variable. For the boosted topology, large-radius jets and substructure techniques are used to identify the decays of boosted $\mathrm{W}$ bosons and top quarks. In this case, the final discriminant is the scalar $\mathrm{p}_{\mathrm{T}}$ sum of all selected jets in an event, $\mathrm{H}_{\mathrm{T}}$. Events are categorized according to the number of b-tagged jets.

An estimation of the dominant QCD multijet background is performed using backgroundrich control regions in data. The observed data agree with the predicted SM background processes. Figure 1 (right) shows the upper limits on the product of the $\mathrm{H}^{ \pm}$associated top quark production cross section and $\mathrm{H}^{ \pm}$branching fraction into a top and bottom quarkantiquark pair.

\section{Search for $\mathrm{H} \rightarrow \mathrm{ZA} \rightarrow \ell^{+} \ell^{-} \mathrm{b} \overline{\mathrm{b}}$}

In the 2HDM alignment limit, and assuming twisted custodial symmetry, one of the largest branching fractions for the heavy scalar $\mathrm{H}$ is for the decay to a lighter pseudoscalar $\mathrm{A}$ and a $\mathrm{Z}$ boson. In this context a search is performed for $\mathrm{H} \rightarrow \mathrm{ZA}$ where the $\mathrm{Z}$ decays into an opposite-sign electron or muon pair, and the light Higgs boson into a b quark pair [8].

The invariant mass distributions of the $\ell \ell \mathrm{b} \bar{b}$ and $\mathrm{b} \bar{b}$ systems, $m_{\ell \ell j \mathrm{j}}$ and $\mathrm{m}_{\mathrm{jj}}$, are used to search for a resonant-like excess compatible with the $\mathrm{H}$ and $\mathrm{A}$ bosons. As these distributions 

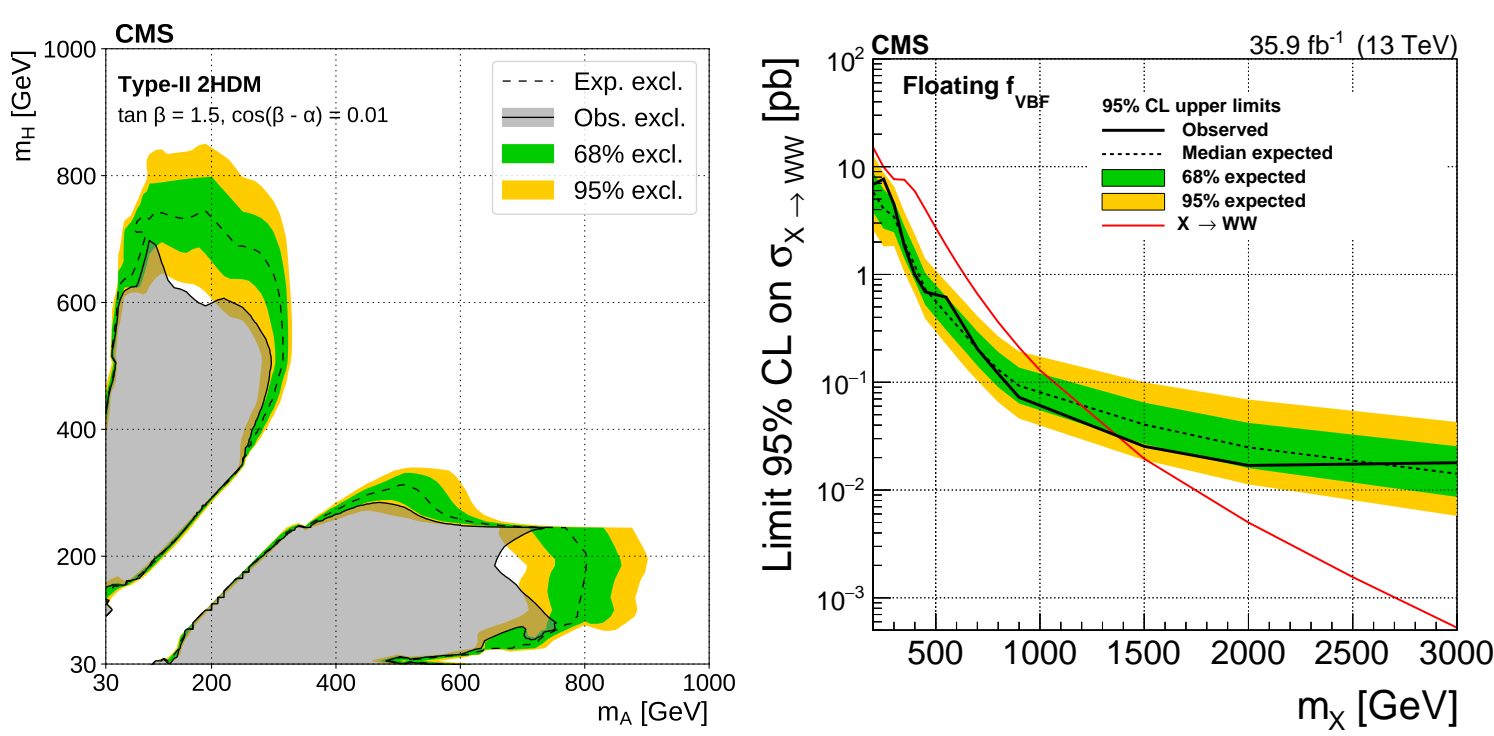

Figure 2: Exclusion limits in the $\mathrm{m}_{\mathrm{A}}-\mathrm{m}_{\mathrm{H}}$ plane for a $2 \mathrm{HDM}$ benchmark with $\tan \beta=1.5$ and $=$ 0.01 (left) [8], and upper limits on the heavy Higgs boson cross section times branching fraction to two $\mathrm{W}$ bosons (right) [11].

are inherently positively correlated, an elliptical signal region in the $\mathrm{m}_{\ell \ell \mathrm{jj}}-\mathrm{m}_{\mathrm{jj}}$ plane is chosen. Selected events in the $m_{\ell \ell j \mathrm{j}}-\mathrm{m}_{\mathrm{jj}}$ plane are classified in six regions around the center of the ellipse, which is seperatley defined for each signal hypothesis.

Two categories are defined based on the flavour of the lepton pairs. The dominant $\mathrm{Z}+$ jets background process is estimated from NLO simulation, while a different-flavour lepton pair control region is used to estimate the top quark background. No significant deviations from the SM expectations are observed. Figure 2 (left) shows the exclusion limits in the $\mathrm{m}_{\mathrm{A}}-\mathrm{m}_{\mathrm{H}}$ plane for a $2 \mathrm{HDM}$ benchmark with $\tan \beta=1.5$ and $=0.01$. Limits are also set for the case of a non-twisted custodial symmetry scenario, where the masses of the $\mathrm{A}$ and $\mathrm{H}$ bosons are exchanged.

\section{Search for $\mathrm{H} \rightarrow \mathrm{WW}$}

A search for a heavy Higgs boson decaying to a pair of $\mathrm{W}$ bosons in the mass range from $200 \mathrm{GeV}$ to $3 \mathrm{TeV}$ is presented [11]. The decay of the $\mathrm{W}$ boson pair is reconstructed in the $2 \ell 2 \nu$ and $\ell \nu 2 \mathrm{q}$ final states. Both gluon fusion and vector boson fusion production of the signal are considered, with a number of hypotheses for their relative contribution considered. Interference effects between the signal and background are also taken into account. Dedicated event categorizations based on the kinematic properties of associated jets and matrix element techniques are employed to optimise the signal sensitivity.

For the semi-leptonic channel, the hadronically decaying $\mathrm{W}$ boson may be reconstructed as two quark-jets (a dijet) or as a single boosted large-radius jet. The final discriminating variable is the invariant mass of the WW system. Both the same flavour and different flavour final states are considered in the fully leptonic channel. The final discriminating 

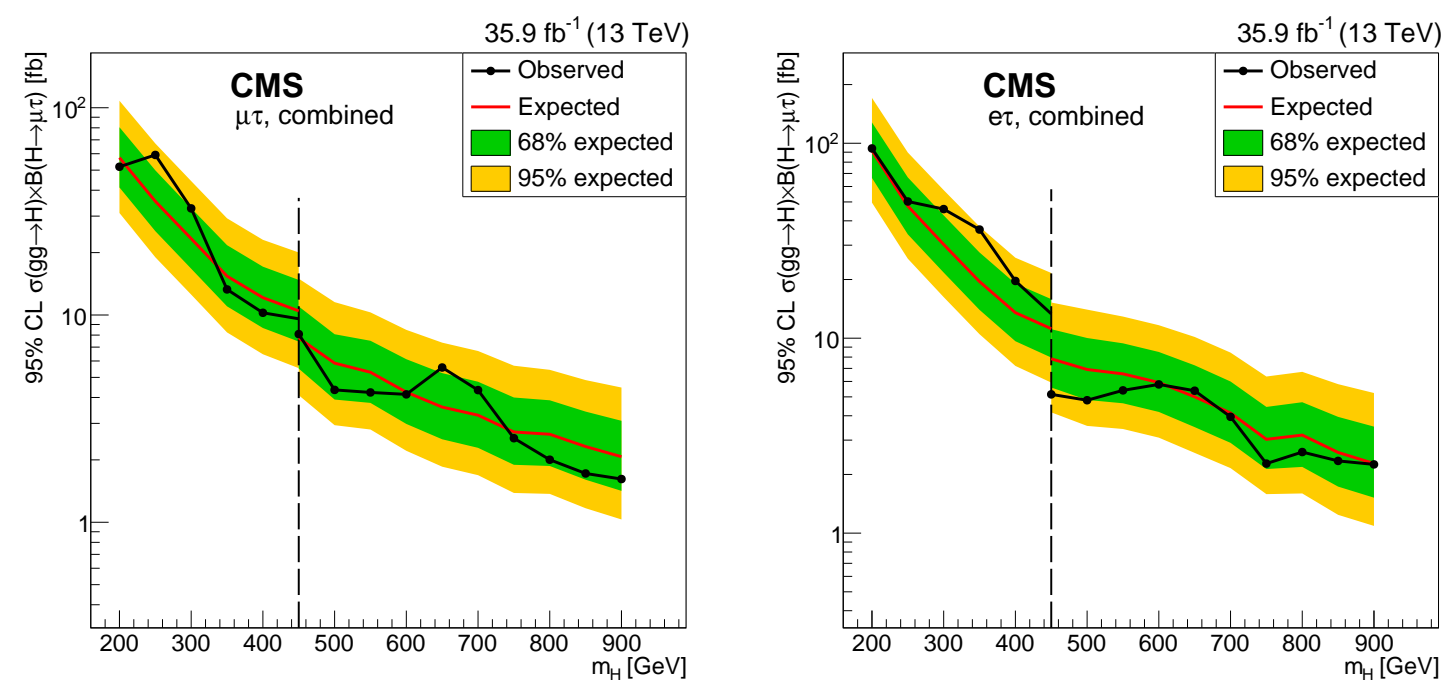

Figure 3: Upper limits on the heavy Higgs boson production cross section times branching ratio to LFV lepton pairs $\mu \tau$ (left) and $e \tau$ (right) [10].

variable used is the visible Higgs mass reconstructed with the dilepton four-momentum and the measured missing momentum in the event. The dominant backgrounds are from nonresonant WW, $\mathrm{W}+$ jets, top quark and Drell-Yan production depending on the category.

The dominant backgrounds are modelled by simulation with their normalisations determined using control regions in data. No evidence for an excess of events with respect to the SM predictions is observed. Figure 2 (right) shows the upper limits on the heavy Higgs boson cross section times branching fraction to two $\mathrm{W}$ bosons, the fraction of the VBF production cross section with respect to the total cross section has been allowed to float in the fit to the data.

\section{Search for lepton flavour violating $H \rightarrow \mu \tau$ and $H \rightarrow e \tau$}

A search has been performed for lepton flavor violating (LFV) decays of a heavy Higgs boson in the $\mu \tau_{h}, \mu \tau_{e}, e \tau_{h}$ and $e \tau_{\mu}$ channels [10], where $\tau_{h}, \tau_{e}$ and $\tau_{\mu}$ correspond to the hadronic, electronic and muonic decay channels of $\tau$ leptons, respectively. The collinear mass of the Higgs boson candidate is determined assuming that, since $\mathrm{m}_{\mathrm{H}}>>\mathrm{m}_{\tau}$, the $\tau$ lepton decay products are highly Lorentz boosted in the direction of the $\tau$ candidate, therefore the neutrino momenta can be approximated to have the same direction as the other visible decay products. The final selection criteria are optimized separately for each decay mode in the $\mathrm{m}_{\mathrm{H}}$ ranges $200<\mathrm{m}_{\mathrm{H}}<450 \mathrm{GeV}$ and $450<\mathrm{m}_{\mathrm{H}}<900 \mathrm{GeV}$. Events are then divided into 0 -jet and 1-jet categories according to the number of jets in the event. The most significant backgrounds come from $\mathrm{W}+$ jets and QCD multijet production, where one or two jets are misidentified as leptons. The misidentified lepton probability is estimated from $\mathrm{Z}+$ jets events in data. 
A maximum likelihood fit is performed to the collinear mass distributions and no significant excess over the background-only hypothesis is observed in any category. Figure 3 shows the resulting upper limits on the heavy Higgs boson production cross section times branching ratio to LFV lepton pairs.

\section{Summary}

A number of searches for additional Higgs bosons have been carried out by the CMS experiment using 2016 data at a center-of-mass energy of $13 \mathrm{TeV}$. No evidence for beyondthe-SM physics has been observed. Upper limits on the Higgs boson production cross section times branching ratio are set for each search. These results further reduce the allowed parameter space for extensions of the standard model.

\section{References}

[1] ATLAS Collaboration, Observation of a new particle in the search for the standard model Higgs boson with the ATLAS detector at the LHC, Phys. Lett. B 716 (2012) 1.

[2] CMS Collaboration, Observation of a new boson at a mass of $125 \mathrm{GeV}$ with the CMS experiment at the LHC, Phys. Lett. B $\mathbf{7 1 6}$ (2012) 30.

[3] CMS Collaboration, Observation of a new boson with mass near $125 \mathrm{GeV}$ in pp Collisions at $\sqrt{s}=7$ and 8 TeV, JHEP 06 (2013) 081.

[4] Branco, G. C. and Ferreira, P. M. and Lavoura, L. and Rebelo, M. N. and Sher, Marc and Silva, Joao P., Theory and phenomenology of two-Higgs-doublet models, Phys. Rept. 516 (2012) 1.

[5] Fayet, Pierre, Supergauge invariant extension of the Higgs mechanism and a model for the electron and its neutrino, Nucl. Phys. B 90 (1975) 104.

[6] CMS Collaboration, The CMS experiment at the CERN LHC, JINST 3 (2008) S08004.

[7] CMS Collaboration, Search for a light charged Higgs boson in the $\mathrm{H}^{ \pm} \rightarrow$ cs channel in proton-proton collisions at $\sqrt{s}=13 \mathrm{TeV}$, Phys. Rev. D 102 (2020) 072001.

[8] CMS Collaboration, Search for 2HDM neutral Higgs bosons through the $\mathrm{H} \rightarrow \mathrm{ZA} \rightarrow$ $\ell^{+} \ell^{-} \mathrm{b} \overline{\mathrm{b}}$ process in proton-proton collisions at $\sqrt{s}=13 \mathrm{TeV}$, JHEP $\mathbf{3}$ (2020) 055.

[9] CMS Collaboration, Search for charged Higgs bosons decaying into a top and a bottom quark in the all-jet final state of pp collisions at $\sqrt{s}=13 \mathrm{TeV}$, JHEP 7 (2020) 126.

[10] CMS Collaboration, Search for lepton flavour violating decays of neutral heavy Higgs boson to $\mu \tau$ and e $\tau$ in proton-proton collisions at $\sqrt{s}=13 \mathrm{TeV}$, JHEP 3 (2020) 103.

[11] CMS Collaboration, Search for a heavy Higgs boson decaying to a pair of $W$ bosons in proton-proton collisions at $\sqrt{s}=13 \mathrm{TeV}$, JHEP 03 (2019) 034. 